\title{
Cubozoa e Scyphozoa (Cnidaria: Medusozoa) de águas costeiras do Brasil
}

\author{
André C. Morandini ${ }^{1}$, Denise Ascher ${ }^{2}$, Sergio N. Stampar ${ }^{1} \&$ João Fernando V. Ferreira ${ }^{3}$ \\ 1. Departamento de Zoologia, Instituto de Biociências, Universidade de São Paulo, Rua do Matão, trav. 14, n. 101, 05508-900 São \\ Paulo, SP, Brasil. (acmorand@usp.br; stampar@ib.usp.br) \\ 2. Faculdades Integradas Hebraico-Brasileiras Renascença, Rua Prates, 790, Bom Retiro, 01121-000 São Paulo, SP, Brasil. \\ (ascherde@ hotmail.com) \\ 3. Instituto Oceanográfico de Santos, Avenida Almirante Saldanha da Gama, 89, 11030-400 Santos, SP, Brasil. (joaocean@yahoo.com.br)
}

\begin{abstract}
Cubozoa and Scyphozoa (Cnidaria: Medusozoa) from Brazilian coastal waters. Coastal species of Cubozoa and Scyphozoa occurring in Brazil are described, based on museum specimens and recently collected ones. Identification keys and a glossary are also presented. The listed species are: Aurelia sp.; Cassiopea xamachana Bigelow, 1892; Chiropsalmus quadrumanus (Müller, 1859); Chrysaora lactea Eschscholtz, 1829; Drymonema dalmatinum Haeckel, 1880; Linuche unguiculata (Swartz, 1788); Lychnorhiza lucerna Haeckel, 1880; Nausithoe aurea Silveira \& Morandini, 1997; Phyllorhiza punctata von Lendenfeld, 1884; Stomolophus meleagris Agassiz, 1862; Tamoya haplonema Müller, 1859; and Tripedalia cystophora Conant, 1897.
\end{abstract}

KEYWORDS. Scyphozoa, Cubozoa, Cnidaria, identification keys, Brazil.

RESUMO. As espécies de Cubozoa e Scyphozoa costeiras que ocorrem no Brasil são descritas, com base em espécimes de coleções de museus e exemplares recém-coletados. Chaves de identificação e um glossário também são apresentados. As espécies descritas são: Aurelia sp.; Cassiopea xamachana Bigelow, 1892; Chiropsalmus quadrumanus (Müller, 1859); Chrysaora lactea Eschscholtz, 1829; Drymonema dalmatinum Haeckel, 1880; Linuche unguiculata (Swartz, 1788); Lychnorhiza lucerna Haeckel, 1880; Nausithoe aurea Silveira \& Morandini, 1997; Phyllorhiza punctata von Lendenfeld, 1884; Stomolophus meleagris Agassiz, 1862; Tamoya haplonema Müller, 1859 e Tripedalia cystophora Conant, 1897.

PALAVRAS-CHAVE. Scyphozoa, Cubozoa, Cnidaria, chaves de identificação, Brasil.

As medusas (águas-vivas ou mães-d'água) das classes Scyphozoa e Cubozoa (filo Cnidaria) são animais conspícuos do plâncton gelatinoso (CoRNelius, 1997a). As medusas de cifozoários e cubozoários são organismos de grande importância ecológica, econômica e médica, tendo papel relevante nas cadeias alimentares marinhas, afetando atividades pesqueiras e turísticas (ver revisão em Purcell et al., 2001) e podendo causar acidentes envolvendo banhistas e mergulhadores (HADDAD JR. et al., 2002).

Existem cerca de 200 espécies de cifozoários no mundo (Mianzan \& Cornelius, 1999), com 22 registros para o Brasil. Por outro lado, cerca de apenas 20 espécies de cubozoários foram descritas mundialmente, sendo quatro delas registradas para a costa brasileira (MIANZAN \& Cornelius, 1999; Morandini, 2003).

Migotтo et al. (2002) produziram uma lista das espécies de Cnidaria Medusozoa registradas para o Brasil com as respectivas referências. Posteriormente, MARQUES et al. (2003) discutiram a distribuição dos grupos ao longo do litoral brasileiro com base no trabalho de Migotto et al. (2002). Apesar de algumas espécies de cubo- e cifomedusas serem amplamente distribuídas na costa brasileira, nenhum trabalho reúne informações sobre a diversidade de espécies, suas principais características, suas distribuições, ou sequer uma chave para a identificação das mesmas. Alguns autores propuseram chaves de identificação para os grupos: NAUMOV (1961 costa da extinta URSS); LARSON (1976 - nordeste dos EUA); SHIH (1977 - costa atlântica do Canadá); O'SulLIVAN
(1982 - Oceano Glacial Antártico); RYLAND (1995 - noroeste da Europa); Connelius (1997b - chave para todos os gêneros); Heeger (1998 - algumas espécies do Mar Mediterrâneo e Indo-Pacífico); Mianzan \& CoRnelius (1999 - Atlântico sul); SHANGwu et al. (2002 - costa da China e adjacências).

Apesar de encontrarmos no Brasil aproximadamente $10 \%$ da diversidade atualmente reconhecida para os cifozoários e cerca de $20 \%$ de cubozoários, a maioria das espécies foi registrada poucas vezes e estes registros estão esparsos ao longo do litoral brasileiro. O que existe são apenas os registros das ocorrências sem qualquer informação sobre a biologia dos animais. Diversos ambientes nunca foram explorados, assim eventualmente novas espécies e/ou novos registros podem ocorrer. $\mathrm{O}$ conhecimento do grupo no Brasil está longe de atingir um nível razoável, devido à inexistência de coleções representativas da diversidade, escassez de grupos de pesquisa focados em diferentes aspectos dos animais, e à dificuldade inerente do estudo de animais gelatinosos em condições laboratoriais e no próprio ambiente.

O objetivo deste trabalho é apresentar de forma sintetizada as informações sobre as espécies de Cubozoa e Scyphozoa de águas costeiras brasileiras, através de descrições e informações adicionais (referências, distribuição, dados biológicos). Além disso, são apresentadas chaves de identificação destas espécies e um glossário dos termos comumente utilizados para os grupos. 


\section{MATERIAL E MÉTODOS}

Coletas. Os exemplares observados foram coletados de 1999-2002 na superfície da água, com uma pequena rede de mão; em arrastos de camarão; encalhados na praia; ou com mergulho autônomo. Os materiais são provenientes dos municípios de Cananéia, Guarujá, Ilhabela, Santos, São Sebastião e Ubatuba (SP), e dos litorais dos estados do Espírito Santo, Rio de Janeiro e Santa Catarina. Observaram-se alguns espécimes da coleção de cnidários do Museu Nacional da Universidade Federal do Rio de Janeiro (MNRJ) e das coleções didáticas do Departamento de Zoologia da Universidade de São Paulo e da Universidade Federal do Ceará. Parte do material coletado foi depositado na coleção de Cnidaria do Museu de Zoologia da Universidade de São Paulo (MZSP) e estes são mencionados nas descrições das espécies. As siglas ACM, DA, SNS e JFVF referem-se às abreviaturas dos nomes dos autores.

Descrições. A classificação dos grupos foi adaptada de Kramp (1961) e Mianzan \& Cornelius (1999). Os Stauromedusae não foram considerados como parte dos Scyphozoa (segundo MARQues \& Collins, 2004). Para as descrições das espécies utilizaram-se dados das descrições de espécimes do Brasil, das descrições originais, e de espécimes vivos e preservados; o padrão de coloração refere-se apenas aos animais vivos ou recémcoletados, uma vez que as cores rapidamente desaparecem em animais preservados. As medidas morfológicas de estefanocifístomas de Nausithoe aurea seguiram a nomenclatura de JARMS (1991) e JARMS et al. (2002), e foram realizadas sob estereomicroscópio; as medusas foram medidas com uma régua plástica.

Chaves. Neste trabalho estão restritas aos animais que podem ser encontrados encalhados nas praias e em águas costeiras do litoral do Brasil (até aproximadamente $1 \mathrm{~km}$ da costa): registros em águas profundas são apresentados por MORANDINI (2003). Não se incluíram todas as espécies listadas por MigotTo et al. (2002), uma vez que algumas delas não foram coletadas próximas à costa: Pelagia noctiluca (Forskål, 1775); ou são espécies de águas profundas: Atolla spp., Periphylla periphylla (Péron \& Lesueur, 1810); ou, ainda, apresentam registros considerados duvidosos: Catostylus cruciatus (Lesson, 1830), Chrysaora plocamia (Lesson, 1830) e Nausithoe punctata Kölliker, 1853. Também não foram incluídas espécies incertae sedis: Tessera gemmaria Goy, 1979 e Tetraplatia volitans Busch, 1851; nem o representante da ordem Stauromedusae: Kishinouyea corbini Larson, 1990.

$\mathrm{Na}$ chave para medusas de Scyphozoa foram incluídas três espécies (Cassiopea xamachana, Linuche unguiculata e Nausithoe aurea) cujos registros remetem apenas à fase polipóide do ciclo de vida, por se acreditar que a fase medusóide dessas espécies poderá ser encontrada na natureza (espécies marcadas com “*”). Medusas dessas espécies foram observadas a partir de cultivo em laboratório. Elaborou-se uma chave para as espécies de pólipos encontrados no infralitoral raso (até $20 \mathrm{~m}$ de profundidade) que inclui as espécies mencionadas acima; também não foram incluídas todas as espécies de pólipos listadas para o Brasil pois não foram identificadas até espécie (Nausithoe sp.) e outras por serem consideradas grupos de espécies: Stephanoscyphistoma corniformis (Komai, 1936) e S. simplex (Kirkpatrick, 1890) (viz. JARMS, 1990).

Glossário. Foi elaborado utilizando-se como base o glossário apresentado por Mianzan \& Cornelius (1999), também servindo como referência os trabalhos de Mayer (1910), Russell (1970), Arai (1997), Cornelius (1997b) e HADDAD (2002) e a versão em português do livro-texto de BARNES (1990). O intuito deste glossário é padronizar, em língua portuguesa, os termos aplicados aos cifozoários e cubozoários. Após cada termo em português é apresentada a palavra em inglês (retirada de Mianzan \& CoRnelius, 1999). As generalidades sobre os termos em português foram consultadas em FERREIRA (1986). Sugere-se a consulta às seguintes ilustrações: figuras 1 e 2 de CoRnelius (1997b: 118-119); figura 1 de MianZan \& CoRnelius (1999: 515-516); e figuras 19, 20, 21 e 22 de HADDAD (2002: 38, 40-41, 43).

\section{RESULTADOS E DISCUSSÃO}

Filo Cnidaria Verrill, 1865

Subfilo Medusozoa Petersen, 1979 (Tesserazoa Salvini-Plawen, 1978)

Classe Cubozoa Werner, 1975

Ordem Cubomedusae Haeckel, 1880

Família Carybdeidae Gegenbaur, 1856

Gênero Tamoya Müller, 1859

Tamoya haplonema Müller, 1859

(Fig. 1)

Tamoya haplonema MüLLER, 1859:1.

Referências para o Brasil. MÜLLER (1859); VANNUCCI (1957); Mianzan \& Cornelius (1999); Migotto et al. (2002); (A. C. Morandini e A. C. Marques, dados não publicados).

Descrição. Refere-se ao maior exemplar (MZSP 336). Umbrela cúbica, alongada, com bordas arredondadas, mais alta que larga, altura $14 \mathrm{~cm}$, largura $5,5 \mathrm{~cm}$. Superfície exumbrelar lisa. Velário $2 \mathrm{~cm}$ de altura, canais ramificados numerosos. Quatro ropálios perradiais laterais, $3,5 \mathrm{~cm}$ da margem, nichos ropaliares abertos horizontalmente. Quatro pedálios inter-radiais simples (altura $5,5 \mathrm{~cm}$ ), um tentáculo por pedálio. Canal do pedálio com projeção superior (divertículo), curvado na direção do sino. Manúbrio curto com quatro lábios. Facelas inter-radiais verticalmente dispostas. Gônadas vestigiais. Umbrela transparente, tentáculos com bandas violeta (esbranquiçadas quando preservadas).

Material examinado. BRASIL, São Paulo: Ubatuba, jovem, 17.VIII.2002, D.A. \& J.F.V.F. col. (MZSP 0336); Santos, 08.VIII.2002, D.A. \& J.F.V.F. col. (MZSP 366); Cananéia, jovem, 15.III.2002, A.C.M. col. (MZSP 337).

Distribuição. Costa atlântica das Américas, Congo, Guiné, África do Sul, corrente de Bengala. O registro mais ao sul é dado para a costa da Argentina (PASTORINo, 2001).

Comentários. O exemplar coletado em Cananéia (MZSP 337) é o menor da espécie já encontrado no litoral brasileiro. A espécie é rara, porém pode causar acidentes graves (HADDAD JR. et al., 2002), (A. C. Morandini e A. C. Marques, dados não publicados). 
Gênero Tripedalia Conant, 1897

Tripedalia cystophora Conant, 1897

(Fig. 2)

Tripedalia cystophora CONANT, 1897:5.

Referências para o Brasil. Morandini \& MARQuEs (1997); Mianzan \& Cornelius (1999); Migotto et al. (2002); (A. C. Marques, A. C. Morandini e M. M. Pinto, dados não publicados).

Descrição. Umbrela cúbica, altura 8-11 mm, largura 9,75-16 mm. Quatro ropálios perradiais 1,9-2,5 $\mathrm{mm}$ da margem inferior. Doze pedálios, lateralmente comprimidos, altura 3,2-4 mm, largura 0,9-1 mm, um tentáculo por pedálio. Tentáculos com anéis transversais. Canal do pedálio sem divertículo. Velário 1,5-1,75 $\mathrm{mm}$ de altura, seis canais velares não ramificados por quadrante. Quatro frênulas perradiais, comprimento 1,75-2 $\mathrm{mm}$. Ovos no espaço gastrovascular.

Material examinado. BRASIL, Pará: Salinópolis (manguezal foz do rio Sampaio), 3 fêmeas, 12.VII.1986, F.C. Rebello col. (MNRJ 1025). Japão.

Distribuição. Norte do Brasil, Caribe (Jamaica),

Comentários. WERNER et al. (1971) descreveram o ciclo de vida da espécie. WERNER (1983) forneceu uma descrição detalhada da metamorfose do pólipo em medusa. StewART (1996) estudou o comportamento da espécie na natureza.

Família Chirodropidae Haeckel, 1880

Gênero Chiropsalmus Agassiz, 1862

Chiropsalmus quadrumanus (Müller, 1859)

(Fig. 3)

Tamoya quadrumana MüLLER, 1859:1.

Chiropsalmus quadrumanus: Agassiz, 1862:174.

Referências para o Brasil. MÜLLER (1859); VANNUCCI (1954; 1957); Mianzan \& Cornelius (1999); Migotto et al. (2002).

Descrição. Umbrela cúbica, bordas arredondadas, tão alta quanto larga, altura 5,5-9 cm, largura $6,5-12 \mathrm{~cm}$. Exumbrela com pequenas verrugas marrons (animais vivos). Velário 1-1,5 cm de altura, numerosos canais ramificados. Quatro ropálios perradiais laterais, $2-2,5 \mathrm{~cm}$ da margem, nichos ropaliares triangulares. Quatro pedálios palmados inter-radiais (altura $6 \mathrm{~cm}$ ) com seis ou sete tentáculos, cada tentáculo inserido em uma projeção do pedálio. Canal do pedálio com projeção superior ligeiramente convexa. Manúbrio com quatro lábios. Estômago amplo, quatro bolsas perradiais aparentando folhas de um trevo, duas estruturas digitiformes lisas por bolsa, projetando-se na cavidade subumbrelar. Gônadas desenvolvidas. Espécimes translúcidos, exceto pelas verrugas marrons.

Material examinado. BRASIL, São Paulo: Ubatuba, 2 jovens, 28.VI.2002, D.A. \& J.F.V.F. col. (MZSP 338); jovem, 17.VIII.2002, D.A. \& J.F.V.F. col. (MZSP 339).

Distribuição. Costa atlântica das Américas.

Comentários. A espécie é bastante comum no litoral brasileiro, sendo encontrada em quase todos os estados. Em geral é capturada com redes de arrasto. Também está associada a acidentes (HADDAD JR. et al., 2002).

Classe Scyphozoa Goette, 1887

Ordem Coronatae Vanhöffen, 1892

Família Linuchidae Haeckel, 1880

Gênero Linuche Eschscholtz, 1829

Linuche unguiculata (Swartz, 1788)

(Figs. 4, 5)

Medusa unguiculata SWARTZ, 1788:199.

Linuche unguiculata; EschscholTz, 1829:91.

Linerges mercurius HAECKEL, 1880:495.

Linerges pegasus HAECKEL, 1880:495.

Liniscus ornithopterus HAECKEL, 1880:497.

Liniscus sandalopterus HAECKEL, 1880:497.

Linuche vesiculata HAECKEL, 1880:499.

Stephanoscyphus komai Leloup, 1937:66 (pólipo).

Referências para o Brasil. SiLveIRA \& MorandinI (1998a; 1998b); MiAnZAN \& CoRnelius (1999); MigotTo et al. (2002).

Descrição. Pólipo: pólipos coloniais, conectados por ciforriza estolonal. Coloração do tubo peridérmico marrom claro. Tubo com nenhum a dois espinhos internos, em forma de xícara com espinhos secundários na borda. Tubo isodiamétrico. Muitas zooxantelas na gastrodeme. Medusa (cultivada em laboratório): medusas pequenas, altura $20 \mathrm{~mm}$, umbrela lisa, forma de dedal, topo do sino achatado e sulco coronal logo abaixo, grande quantidade de zooxantelas. Lóbulos marginais sobrepostos em seqüência. Oito tentáculos ad-radiais pequenos. Manúbrio com quatro lábios recurvados. Oito gônadas alongadas, forma crescente, em quatro pares.

Material examinado. BRASIL, Rio de Janeiro: Parati (Ilha dos Ganchos), 24.IV.2002, S.N.S. col. (MZSP 462); Santa Catarina: Ilha do Arvoredo $\left(27^{\circ} 17,50^{\prime}\right.$ 'S - $\left.48^{\circ} 22^{\prime} \mathrm{W}\right), 28$ 29.V.2001 e 14.II.2002, A.C.M. col. (MZSP 463).

Distribuição. Atlântico tropical ocidental (Caribe), África ocidental (Serra Leoa), Indo-Pacífico tropical. No Brasil, estado de São Paulo; novos registros para os estados de Santa Catarina e Rio de Janeiro.

Comentários. Os pólipos foram descritos como uma outra espécie por Leloup (1937). No Brasil a espécie é encontrada sempre associada a regiões de substrato de origem calcária. Na literatura comenta-se que a cavidade subumbrelar possui quarenta e oito estruturas saciformes, arranjadas em três verticilos (o mais externo com trinta e dois, o mediano com oito e o mais interno também com oito sacos) (MAYER, 1910); tal característica não foi observada nas medusas cultivadas.

Família Nausithoidae Haeckel, 1880

Gênero Nausithoe Kölliker, 1853

Nausithoe aurea Silveira \& Morandini, 1997

(Figs. 6, 7)

Nausithoe aurea SilveIRA \& MoRANDINI, 1997:236.

Referências para o Brasil. SiLveIRA \& MoRANDinI (1997); Morandini \& Silveira (2001); Migotto et al. (2002); SILVEIRA et al. (2003). 

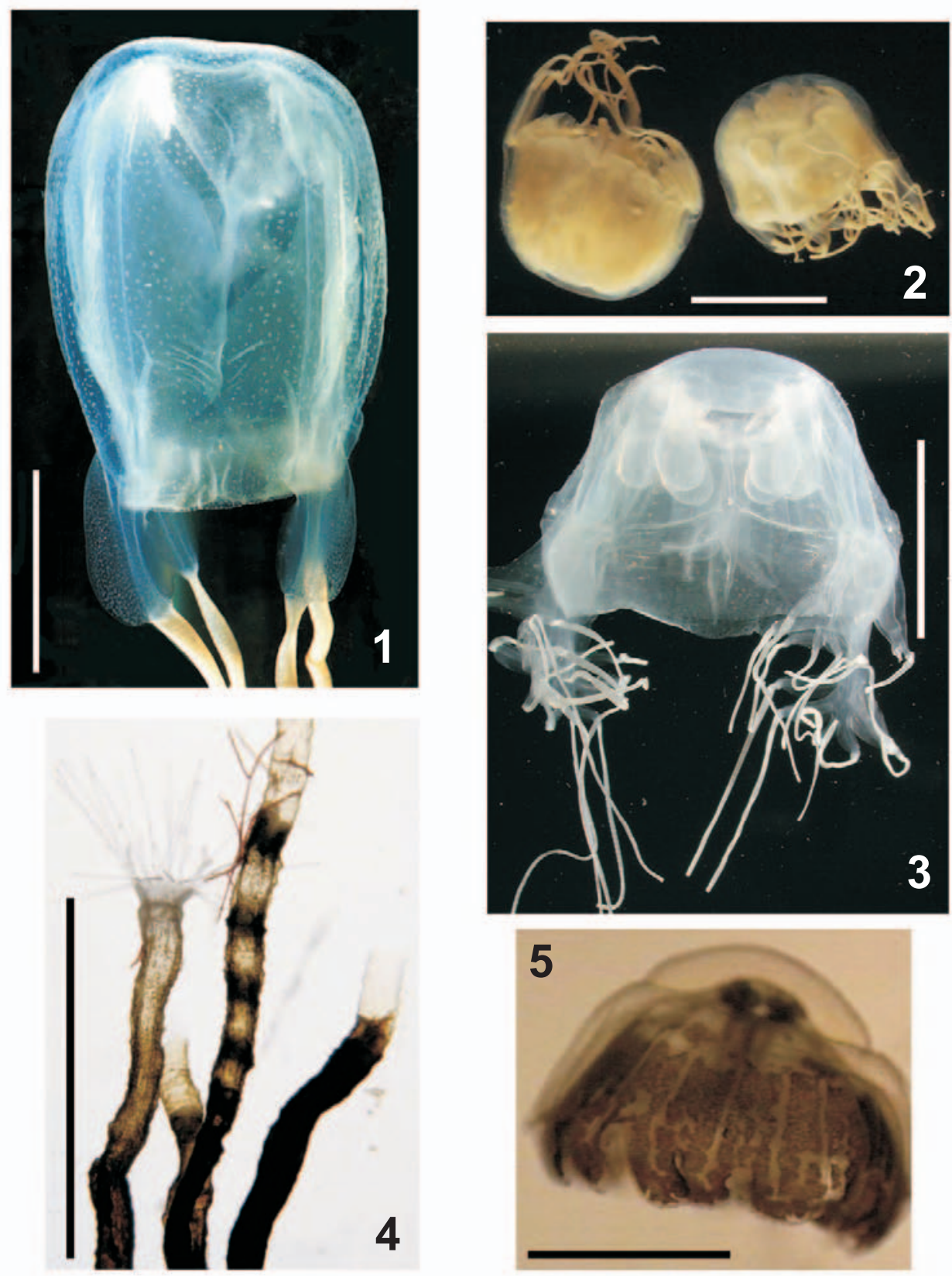

Figs. 1-5. 1, Tamoya haplonema Müller, 1859, exemplar vivo com apenas um tentáculo inserido em cada pedálio, fotografado em aquário no CEBIMar-USP (Foto: Alvaro E. Migotto); 2, Tripedalia cystophora Conant, 1897, exemplares preservados com mais de um pedálio em cada canto da umbrela; 3, Chiropsalmus quadrumanus (Müller, 1859), exemplar vivo fotografado em aquário no CEBIMar-USP; observar pedálio palmado e projeções digitiformes, por transparência, na subumbrela (Foto: Alvaro E. Migotto); 4, pólipos de Linuche unguiculata (Swartz, 1788), colônia viva com tubos quase que isodiamétricos e pólipo central iniciando estrobilização 5 , medusa de Linuche unguiculata (Swartz, 1788), exemplar vivo com sulco coronal na exumbrela e grande quantidade de zooxantelas, cultivado em laboratório (Foto digitalizada a partir de VHS: Fábio L. da Silveira). Escalas: Figs. 1, 3, 5 cm; Fig. 2, 1,5 cm; Fig. 4, 1 cm; Fig. $5,0,5 \mathrm{~cm}$.

Descrição. Pólipo: cifístomas solitários, tubo peridérmico 1,4-9,18 mm comprimento, marrom claro a escuro, pequeno disco basal de fixação (diâmetro 0,19$0,85 \mathrm{~mm}$ ). Tubo peridérmico com séries transversais de 16 espinhos internos. Manchas pigmentares brancas na base dos tentáculos. Formquotient a $2 \mathrm{~mm}$ de altura 0,120,31 e a $5 \mathrm{~mm}$ de altura $0,07-0,23$. Medidas morfológicas dos pólipos dos estados do Espírito Santo e Santa
Catarina são apresentadas na tabela I. Medusa (cultivada em laboratório): medusas pequenas, transparentes, 4,8$10,5 \mathrm{~mm}$ diâmetro, umbrela lisa, achatada. Oito tentáculos, 5,5 mm comprimento. Manúbrio curto. Dezesseis lóbulos marginais com uma mancha pigmentar amarela. Oito gônadas ad-radiais arredondadas, marrons. Filamentos gástricos em quatro grupos de três (eventualmente quatro). Oito ropálios com estatocisto, ocelo vermelho. 
Tabela I. Medidas morfológicas e Formquotient (proporções) de estefanocifístomas de Nausithoe aurea Silveira \& Morandini, 1997 dos estados do Espírito Santo (ES) e Santa Catarina (SC) (logo após a coleta) em comparação com pólipos do estado de São Paulo, Canal de São Sabastião (descr. original e Ago 1997) e estado da Bahia (BA) [Db, diâmetro do tubo logo acima do disco basal; Dbd, diâmetro do disco basal; Do, diâmetro da abertura do tubo; D/L $\mathrm{L}_{2 \mathrm{~mm}}$, Formquotient a $2 \mathrm{~mm}$ de altura $\left(\mathrm{D}_{2 \mathrm{~mm}} / 2\right)$; D/L $\mathrm{L}_{5 \mathrm{~mm}}$, Formquotient a $5 \mathrm{~mm}$ de altura $\left(\mathrm{D}_{5 \mathrm{~mm}} / 5\right)$; D/Ltot, Formquotient (Do/Ltot); Ltot, comprimento total do tubo; descr. original, resumo dos dados da descrição original da espécie (Silveira \& Morandini, 1997); Ago/1997, resumo dos dados de 24 pólipos coletados em agosto de 1997; BA, resumo dos dados de 5 pólipos do estado da Bahia (Morandini \& Silveira, 2001)].

\begin{tabular}{|c|c|c|c|c|c|c|c|}
\hline Pólipo & $\operatorname{Dbd}(\mathrm{mm})$ & $\mathrm{Db}(\mathrm{mm})$ & Ltot $(\mathrm{mm})$ & $\mathrm{D} / \mathrm{L}_{2 \mathrm{~mm}}$ & $\mathrm{D} / \mathrm{L}_{5 \mathrm{~mm}}$ & Do $(\mathrm{mm})$ & D/Ltot \\
\hline ES & 0,66 & 0,22 & 2,5 & 0,2 & & 0,76 & 0,304 \\
\hline ES & 0,5 & 0,16 & 4,22 & 0,23 & & 0,7 & 0,165 \\
\hline ES & 0,62 & 0,22 & 3,17 & 0,18 & & 0,56 & 0,176 \\
\hline $\mathrm{SC}$ & & 0,22 & 5,61 & 0,31 & 0,18 & 0,92 & 0,163 \\
\hline $\mathrm{SC}$ & & 0,52 & 6,34 & 0,31 & 0,14 & 0,86 & 0,135 \\
\hline $\mathrm{SC}$ & & 0,3 & 2,47 & 0,25 & & 0,58 & 0,234 \\
\hline SC & 0,44 & 0,18 & 6,19 & 0,18 & 0,128 & 0,62 & 0,1 \\
\hline SC & 0,42 & 0,18 & 6,82 & 0,25 & 0,236 & 1,0 & 0,146 \\
\hline $\mathrm{SC}$ & 0,44 & 0,16 & 8,25 & 0,22 & 0,12 & 0,74 & 0,089 \\
\hline descr. original & $0,3-0,85$ & $0,12-0,27$ & $1,4-9,18$ & $0,127-0,24$ & $0,07-0,14$ & $0,34-1,02$ & $0,078-0,32$ \\
\hline Ago/1997 & $0,19-0,49$ & $0,09-0,26$ & $2,4-7,54$ & $0,15-0,29$ & $0,10-0,14$ & $0,45-0,85$ & $0,08-0,205$ \\
\hline BA & $0,31-0,51$ & $0,13-0,2$ & $2,7-6,8$ & $0,18-0,24$ & 0,10 & $0,49-0,66$ & $0,09-0,21$ \\
\hline
\end{tabular}

Material examinado. BRASIL, Espírito Santo: Aracruz, 05.II.2002, P. A. Horta Jr. col. (MZSP 464); Rio de Janeiro: Ilha Grande, 24.VII.2000, L. L. Longo col. (MZSP 465); Santa Catarina: Ilha do Arvoredo $\left(27^{\circ} 17,50^{\prime} \mathrm{S}-48^{\circ} 22^{\prime} \mathrm{W}\right), 28$ 29.V.2001 e 14.II.2002, A.C.M. col. (MZSP 466).

Distribuição. Litoral brasileiro, estados do Rio de Janeiro, São Paulo e Bahia; novos registros para os estados do Espírito Santo e Santa Catarina.

Comentários. Até o presente, a espécie é endêmica do litoral brasileiro. Cifístomas estão sempre associados a substrato calcário.

Subclasse Discomedusae Haeckel, 1880

Ordem Semaeostomeae Agassiz, 1862

Família Pelagiidae Gegenbaur, 1856

Gênero Chrysaora Péron \& Lesueur, 1810

\section{Chrysaora lactea Eschscholtz, 1829}

(Figs. 8, 9)

Chrysaora lactea Eschscholtz, 1829:81.

Dactylometra lactea; Agassiz, 1862:26.

Chrysaora hysoscella; VANNUCCI, 1954:123.

Chrysaora quinquecirrha; GoY, 1979:291.

Referências para o Brasil. EschschOLTz (1829); Oliveira (1950); Vannucci (1954; 1957); Goy (1979); Mianzan \& Cornelius (1999); Migotto et al. (2002); MoRANDini et al. (2004).

Descrição. Umbrela ligeiramente achatada a quase hemisférica, diâmetro 6,5-14,5 cm. Exumbrela com minúsculas verrugas irregularmente distribuídas. Tentáculos, 24 a 31, cada octante possuindo três a cinco. Quatro braços orais, pouco espiralados, com bordas franjadas. Bolsas gástricas ropaliares menores que tentaculares. Septos radiais estreitos, terminação interna alargada. Coloração transparente a branco-leitosa, manchas irregulares marrom a vinho, alguns espécimes com dezesseis manchas triangulares radialmente dispostas (Fig. 8).

Material examinado. BRASIL, São Paulo: Ubatuba, 2 jovens, 30.XI.2002, D.A. \& J.F.V.F. col. (MZSP 340); Guarujá, 7 jovens, 10.VIII.2002, D.A. \& J.F.V.F. col. (MZSP 341); Cananéia, 1 jovem, 15.XII.1999, A.C.M. col. (MZSP 342). do Sul.

Distribuição. Jamaica e costa atlântica da América

Comentários. Mianzan (1989a, b) registrou a ocorrência de estágios jovens em amostras de plâncton da costa da Argentina. Tronolone et al. (2002) descreveram o estágio de éfira a partir de material coletado na natureza e compararam com materiais a partir de cultivo em laboratório. MoRANDINI et al. (2004) descreveram o ciclo de vida da espécie.

Família Cyaneidae Agassiz, 1862

Gênero Drymonema Haeckel, 1880

\section{Drymonema dalmatinum Haeckel, 1880}

(Fig. 10)

Drymonema dalmatina HAECKEL, 1880:642.

Drymonema victoria HAECKEL, 1882:125.

Drymonema gorgo MülLER, 1883:221.

Drymonema cordelio ANTIPA, 1892:339.

Drymonema dalmatinum; Kramp, 1961:336.

Referências para o Brasil. MÜLLER (1883); VANNUCCI (1957); Mianzan \& CoRnelius (1999); Migotto et al. (2002).

Descrição. Umbrela ligeiramente achatada, diâmetro até $1 \mathrm{~m}$ (40 e $18 \mathrm{~cm}$ nos espécimes examinados). Numerosos lóbulos marginais alongados, fundidos, formando sulcos. Tentáculos na subumbrela, distantes da margem. Braços orais foliáceos, finos, alongados. Ramos das bolsas gástricas estreitos, na mesma quantidade dos lóbulos. Oito nichos ropaliares subumbrelares, lembrando duas salsichas conectadas pelas pontas. Coloração marrom avermelhada, tentáculos e margem rosados.

Material examinado. BRASIL, São Paulo: Ilhabela (praia do Sino), 23.II.1997, P. F. S. Cornelius \& F. L. da Silveira col. (MZSP 343); Ilhabela (praia do Portinho), 1 jovem, 05.VII.1997, L. P. Andrade \& V. B. Tronolone col. (MZSP 344).

Distribuição. Costa atlântica da América do Sul e Caribe, mares Adriático e Egeu.

Comentários. Estes exemplares foram os únicos a serem registrados após a descrição de MüLlER (1883) (P. F. S. Cornelius e F. L. da Silveira, dados não publicados). 
Família Ulmaridae Haeckel, 1880

Gênero Aurelia Péron \& Lesueur, 1810

Aurelia sp.

(Fig. 11)

Referências para o Brasil. Aurelia aurita: PANTIN \& Dias (1952); VannucCi (1957); Goy (1979); Mianzan \& Cornelius (1999); MigotTo et al. (2002).

Aurelia sp. 2: DAwsOn \& JACOBS (2001); MigOTto et al. (2002).

Descrição. Umbrela achatada, transparente, diâmetro $17-29 \mathrm{~cm}$. Manúbrio muito curto. Oito ropálios. Margem umbrelar danificada, impossibilitando a contagem dos lobos. Braços orais também danificados. Bolsas gástricas e gônadas em forma de ferradura. Tentáculos e gônadas de cor rosa. Dezesseis canais radiais não ramificados a partir do estômago, dois a quatro canais radiais ramificados entre eles.

Material examinado. BRASIL, São Paulo: Ilhabela (praia do Velasques), 1 jovem, 28.II.2002, O. M. P. Oliveira col. (MZSP 345); Cananéia, 2 fêmeas, 19.IV.2000, A.C.M. col. (MZSP 346).

Distribuição. Se considerarmos a espécie como nova, segundo as análises de DAwsON \& JACOBS (2001), a distribuição está restrita ao litoral brasileiro. No entanto, A. aurita é cosmopolita

Comentários. Todos os espécimes estavam danificados, sem algumas estruturas marginais (ropálios, tentáculos, lobos) e sem os braços orais. Os espécimes de Cananéia apresentavam uma morfologia anormal (com simetria trímera ou pentâmera) como observado por Gershwin (1999) em exemplares de Aurelia aurita (Linnaeus, 1758) e Aurelia labiata Chamisso \& Eysenhardt, 1821 cultivados em condições de laboratório. Amostras dos espécimes de Cananéia foram enviadas ao Dr. M. N. Dawson (Austrália), e utilizadas em análises moleculares (DAwson \& JACOBS, 2001), onde os autores concluíram se tratar de uma nova espécie. No entanto, enfatizamos que a ausência de espécimes, em condições razoáveis de estudo, limita a corroboração dos argumentos (nova espécie) dos autores (DAwson \& JACOBS, 2001) com base, também, em características morfológicas. Além disso, a sistemática do gênero Aurelia vem sendo amplamente discutida (DAWSON \& MARTIN, 2001; Schroth et al., 2002; Dawson, 2003), sem nenhum consenso atual sobre o número e a delimitação das espécies.

Ordem Rhizostomeae Cuvier, 1799

Família Cassiopeidae Agassiz, 1862

Gênero Cassiopea Péron \& Lesueur, 1810

Cassiopea xamachana Bigelow, 1892

(Figs. 12, 13)

Cassiopea xamachana Bigelow, 1892:212.

Cassiopea frondosa; FewKes, 1882:254.

Polyclonia xamachana; VANHÖFFEN, 1911:321.

Cassiopea xamachana bonairensis HUMMELINCK, 1933:497.

Cassiopea xamachana tortuguensis HUMMELINCK, 1933:494.

Cassiopea xamachana typica Hummelinck, 1933:494.

Referências para o Brasil. Migotтo et al. (2002).

Descrição. Pólipo: cifístomas com diâmetro do disco oral $0,75-1,8 \mathrm{~mm}$, altura do cálice $0,55-1,1 \mathrm{~mm}$ e altura do pedúnculo 1,75-4 mm e diâmetro do pedúnculo $0,15-0,3$ $\mathrm{mm}$. Tentáculos 29-33, até três vezes a altura do pólipo. Boca cruciforme, lábios proeminentes, elevados em relação ao disco oral. Quatro septos gástricos, quatro óstios entre eles. Formação de planulóides (porção inferior do cálice). Coloração esbranquiçada. Pólipos com zooxantelas. Estrobilização monodisco. Medusa (cultivada em laboratório): umbrela achatada, diâmetro 2,5-7 cm, depressão central na exumbrela. Superfície oral voltada para cima. Cinco lóbulos marginais pequenos, arredondados entre ropálios. Ropálios, 15-16, em nichos protegidos por uma fina membrana. Quatro pares de braços orais ad-radiais, mais longos que o raio, triangulares em corte transversal, superfície aboral chata. Poucas e pequenas vesículas nos braços orais. Canais radiais 30-32 (15-16 ropaliares), distalmente bifurcados, muito ramificados em anastomoses laterais. Zooxantelas na gastroderme, coloração azul acinzentada com manchas brancas alinhadas aos ropálios.

Material examinado. BRASIL, São Paulo: São Sebastião (tanques de cultivo com água corrente - pólipos), 1999, A. E Migotto col. (MZSP 347); São Paulo (cultivo - éfira), 18.IV.2002, F. L. da Silveira \& A.C.M. col. (MZSP 348); São Paulo (cultivo medusa), 18.I.2002, F. L. da Silveira \& A.C.M. col. (MZSP 349). (EUA).

Distribuição. São Sebastião (Brasil); Caribe; Flórida

Comentários. Nenhuma medusa da espécie foi observada no litoral brasileiro. Em laboratório, os pólipos se fixam em plástico ou vidro.

Família Lychnorhizidae Haeckel, 1880

Gênero Lychnorhiza Haeckel, 1880

\section{Lychnorhiza lucerna Haeckel, 1880}

(Fig. 14)

Lychnorhiza lucerna HAECKEL, 1880:587.

Cramborhiza flagellata HAECKEL, 1880:646.

Lychnorhiza flagellata; VANHÖFFEN, 1888:29, 42.

Referências para o Brasil. HaEckel (1880); VannucCi $(1951 ; 1954 ; 1957)$; Mianzan \& CoRnelius (1999); Silveira \& Cornelius (2000); MigotTo et al. (2002).

Descrição. Umbrela hemisférica a ligeiramente achatada, diâmetro 4,5-25 cm. Coloração esbranquiçada, com manchas marrons irregulares, alguns exemplares com lóbulos azul-escuros. Mesogléia flexível. Diminutas verrugas exumbrelares. Lóbulos marginais triangulares, reentrâncias arredondadas, quatro lóbulos interropaliares. Lóbulos ropaliares pequenos, pontudos, com laterais paralelas. Oito ropálios, esbranquiçados, em reentrâncias profundas. Quatro pares de braços orais adradiais, com três abas, lateralmente comprimidos na base, comprimento semelhante ao diâmetro da umbrela. Bocas na porção interna dos braços. Poucos filamentos nos braços. Disco oral (braquial) amplo, com quatro pilares de bases largas, óstios subgenitais duas vezes mais largos que pilares. Estômago cruciforme, com cantos arredondados. Boca central ausente. Canal circular distante 1/3 da margem. Rede de canais anastomosados, externos ao canal circular. Três a quatro canais centrípetos de fundo cego (não alcançando o estômago central) entre os 16 canais radiais. 

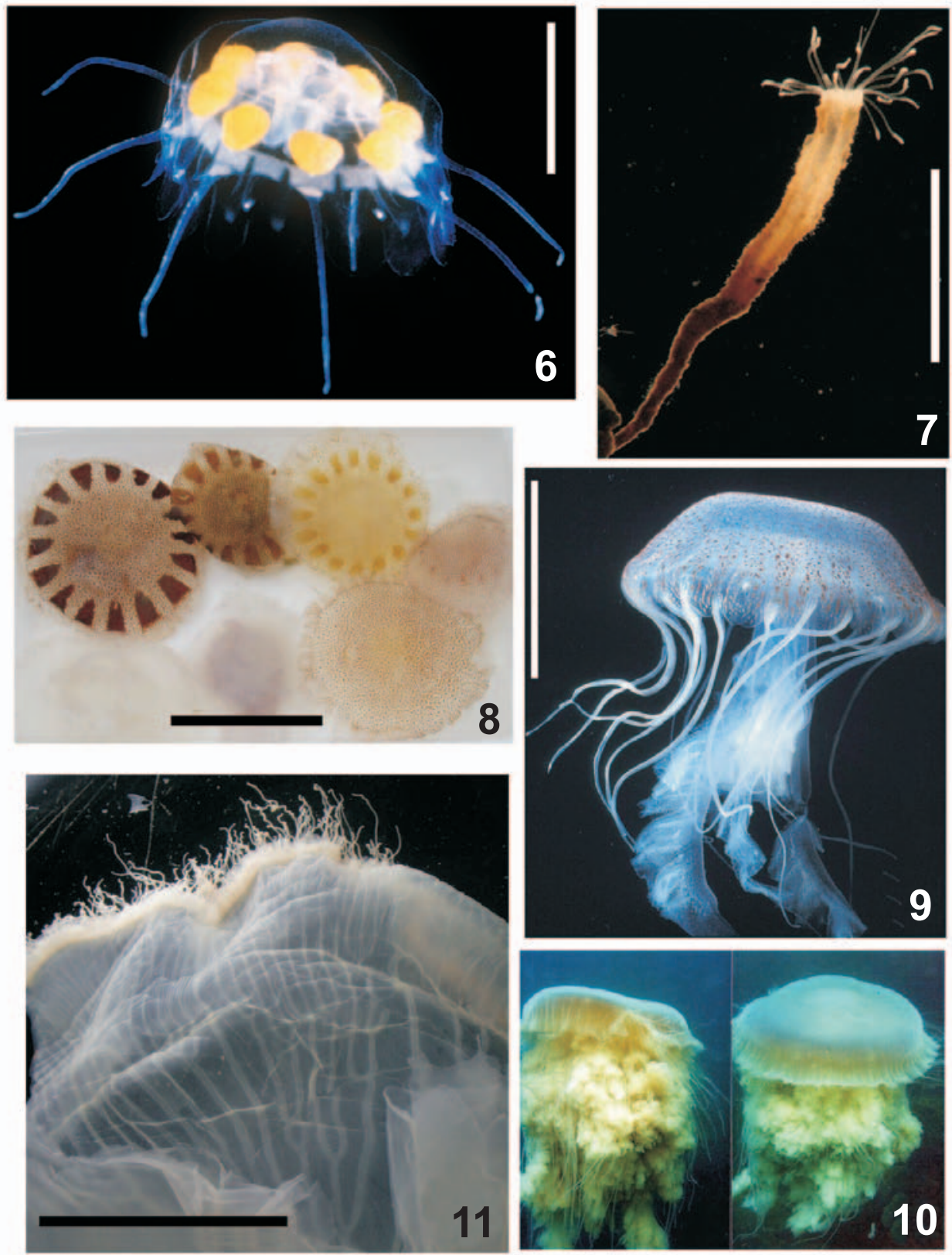

Figs. 6-11. 6, medusa de Nausithoe aurea Silveira \& Morandini, 1997, exemplar vivo cultivado em laboratório e fotografado em aquário (Foto: Alvaro E. Migotto); 7; pólipo de Nausithoe aurea Silveira \& Morandini, 1997, exemplar vivo com hábito solitário e tentáculos parcialmente retraídos; 8, medusas de Chrysaora lactea Eschscholtz, 1829, variação no padrão de coloração de exemplares recém preservados; 9, Chrysaora lactea Eschscholtz, 1829, exemplar vivo fotografado em aquário (Foto: Fábio L. da Silveira); 10, Drymonema dalmatinum Haeckel, 1880, composição de imagens de exemplar vivo, com cerca de $40 \mathrm{~cm}$ de diâmetro, fotografado na natureza; braços orais foliáceos na imagem à esquerda, e aspecto geral do animal à direita (Foto: Maristela Colucci); 11, Aurelia sp, detalhe em vista oral de exemplar preservado com tentáculos marginais e canais do sistema gastrovascular. Escalas: Figs. 6-7, 0,5cm; Figs. 8-9, 11, 8cm.

Espécimes examinados. BRASIL, São Paulo: Ubatuba, 1 jovem, 30.XI.2002, D.A. \& J.F.V.F. col. (MZSP 351); Cananéia, 6 jovens, 15.XII.1999, A.C.M. col. (MZSP 350). Ocidental.

Distribuição. Espécie endêmica do Atlântico Sul

Comentários. A única informação publicada sobre a biologia da espécie é que parasitas (Cestoda) foram encontrados na sua mesogléia (VANNUCCI-MENDES, 1944).
Família Mastigiidae Stiasny, 1921

Gênero Phyllorhiza Agassiz, 1862

Phyllorhiza punctata von Lendenfeld, 1884

(Figs. 15, 16)

Phyllorhiza punctata vON LENDENFELD, 1884:296.

Mastigias scintillae MoreIRA, 1961:6.

Referências para o Brasil. MoreIRA (1961); MiAnZAN 

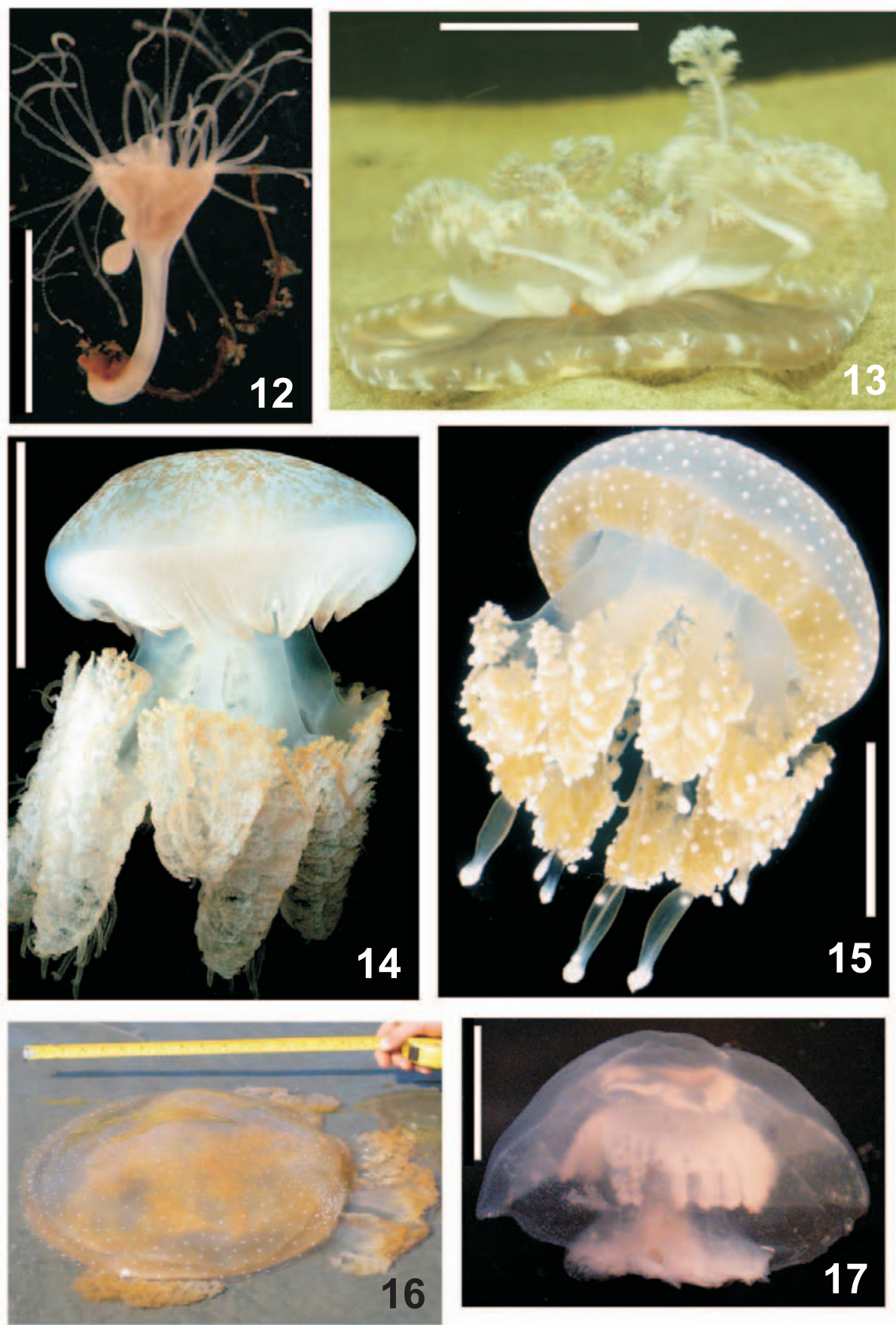

Figs. 12-17. 12, pólipo de Cassiopea xamachana Bigelow, 1892, exemplar vivo com planulóide brotando do cálice do animal; 13 , Cassiopea xamachana Bigelow, 1892, exemplar vivo de medusa obtida e cultivada em laboratório e fotografada em aquário; 14, Lychnorhiza lucerna Haeckel, 1880, exemplar vivo com filamentos nos braços orais, fotografado em aquário no CEBIMar-USP (Foto: Alvaro E. Migotto); 15, Phyllorhiza punctata von Lendenfeld, 1884, exemplar vivo com clavas terminais nos braços orais, fotografado em aquário (Foto: Fábio L. da Silveira); 16, Phyllorhiza punctata von Lendenfeld, 1884, exemplar vivo fotografado encalhado na praia do Perequê (Guarujá, São Paulo) (Foto: Cintia Ferreira); 17, exemplar preservado de Stomolophus meleagris Agassiz, 1862; observar as escapuletas por transparência e a ausência de apêndices nos braços orais fusionados. Escalas: Fig. 12, 0,8 cm; Figs. 13, 15, 17, 3 cm; Fig. 14, $10 \mathrm{~cm}$; Fig. 16, escala amarela aproximadamente $17 \mathrm{~cm}$.

\& Cornelius (1999); Silveira \& Cornelius (2000); MigotTo et al. (2002).

Descrição. Umbrela hemisférica, diâmetro $2,5-26 \mathrm{~cm}$. Exumbrela com elevações proeminentes brancas. Lóbulos marginais truncados ou pontudos, conectados por membrana. Oito ropálios. Musculatura circular interrompida nos canais ropaliares, somente na parte interna ao canal circular. Filamentos esbranquiçados portando larvas na região central do disco braquial. Óstios subgenitais ovais, duas vezes mais largos que 
pilares. Oito braços orais, comprimento 3-7 cm, com filamentos e clavas terminais. Braços orais em forma de "J", com três faces (abas) em cortes transversais. Faces abaxiais com pequenas ramificações e numerosas bocas, ausentes na face interna (adaxial). Estômago central cruciforme, oito canais radiais ropaliares conectados por canal circular interno. Rede externa de canais anastomosados conecta canais ropaliares. Rede interna de canais anastomosados conecta estômago e quatro canais ropaliares perradiais. Coloração marrom, variando de intensidade devido à presença de zooxantelas.

Material examinado. BRASIL, São Paulo: Ubatuba, 4 jovens, 17.VIII.2002, D.A. \& J.F.V.F. col. (MZSP 352); Guarujá, 2 jovens, 09.VIII.2002, D.A. \& J.F.V.F. col. (MZSP 353); Santos, 2 jovens, 09.VII.2002, D.A. \& J.F.V.F. col. (MZSP 367); Cananéia, 7 jovens, 06.II.2002, A.C.M. col. (MZSP 354).

Distribuição. Indo-Pacífico (Austrália, Filipinas, Japão), Atlântico norte (Golfo do México) e sul (Brasil), Mediterrâneo (costa de Israel).

Comentários. A espécie esteve presente no litoral brasileiro em meados da década de 1950, tendo sido descrita como uma nova espécie (MoreIRA, 1961) e pode ter sido transportada artificialmente (P. F. S. Cornelius \& e F. L. da Silveira, dados não publicados). SiLveIRA \& Cornelius (2000) comentam sobre a ocorrência da espécie em diferentes regiões e discutem aspectos de exemplares da espécie encontrados no estado da Bahia.

Família Stomolophidae Haeckel, 1880

Gênero Stomolophus Agassiz, 1862

Stomolophus meleagris Agassiz, 1862

(Fig. 17)

Stomolophus meleagris Agassiz, 1862:138,151.

Stomolophus agaricus HAECKEL, 1880:599.

Stomolophus fritillaria HAECKEL, 1880:598.

Stomolophus chunii VANHÖFFEN, 1888:31,43.

Stomolophus meleagris fritillaria; KRAMP, 1955:165.

Referências para o Brasil. VANNUCCI (1954; 1957); Mianzan \& Cornelius (1999); Migotto et al. (2002).

Descrição. Umbrela mais que um hemisfério, diâmetro 4-6,5 cm (exemplares do Ceará), 11-12 cm (exemplares do Sergipe). Braços orais curtos, fundidos, sem filamentos ou clavas terminais. Mesogléia espessa, rígida. Exumbrela lisa. Lóbulos marginais curtos, arredondados, 20 a 25 por octante. Lóbulos ropaliares pontudos, duas a três vezes mais longos que os demais. Oito ropálios. Musculatura subumbrelar interrompida nos canais radiais. Braços orais fundidos, com abertura bucal central. Escapuletas com bocas, na base de cada braço oral, não se estendendo além da margem do sino. Estômago central pequeno. Canal circular ausente. Canais radiais conectados por rede de canais anastomosados. Coloração esbranquiçada (exemplares preservados).

Espécimes examinados. BRASIL, Ceará: Fortaleza (praia do Meireles), 1990-1992 (UFC); Sergipe: Aracaju (rio Sergipe), 03.I.1997 (MZSP 355).

Distribuição. Costa atlântica das Américas e pacífica da América do Norte.

Comentários. BIGELOw (1914) reconheceu que todas as espécies do gênero deveriam ser consideradas como S. meleagris. Kramp (1955) distinguiu os espécimes do litoral norte da América do Sul como uma variedade distinta (Stomolophus meleagris fritillaria), devido a algumas características morfológicas (tamanho máximo, tamanho das escapuletas e número de lóbulos marginais por octante).

Chave para identificação dos grandes grupos de medusas

1. Umbrela (sino) hemisférica ou discoidal; margem da umbrela recortada; tentáculos, quando presentes, inseridos diretamente na umbrela (marginais ou subumbrelares); ropálio marginal ou subumbrelar

Classe Scyphozoa

Umbrela cúbica; margem da umbrela não recortada; tentáculos inseridos em uma projeção da margem do sino (pedálio); ropálio na exumbrela

Classe Cubozoa

Classe Cubozoa. As medusas pertencentes a este grupo possuem a umbrela em forma cúbica (ou quadrática), com a margem não-recortada. Os animais são geralmente de tamanho grande (maiores que $5 \mathrm{~cm}$ ). As medusas apresentam velário. Os tentáculos são inseridos em projeções da umbrela denominadas pedálios. Um anel nervoso conecta todos os 4 ropálios; os ropálios estão localizados nos lados da exumbrela. O ciclo de vida é metagenético, e a produção de medusas se dá pela metamorfose completa do pólipo.

Chave para identificação das espécies de Cubozoa do Brasil.

1. Um tentáculo por pedálio .......................................... 2 Mais de um tentáculo por pedálio Chiropsalmus quadrumanus

2. Quatro pedálios, um em cada canto do sino Tamoya haplonema

Doze pedálios, formando grupos de três em cada canto do sino Tripedalia cystophora

Classe Scyphozoa. Umbrela circular, com a margem recortada e sem velário. Os tentáculos, quando presentes, diretamente inseridos na umbrela (sino). Na maioria das espécies, os ropálios localizam-se em reentrâncias na margem do sino, e são ladeados por um par de lóbulos, que podem ser diferenciados, denominados lóbulos ropaliares. O ciclo de vida é metagenético, e a produção de medusas ocorre por estrobilização.

Chave para identificação das espécies de Scyphozoa do Brasil (medusas).

1. Medusas com sulco coronal na exumbrela Coronatae 2

Medusas sem sulco coronal na exumbrela Discomedusae .. 3

Ordem Coronatae. As medusas apresentam um sulco coronal dividindo a exumbrela em disco central e região periférica. A umbrela pode variar de achatada a um domo elevado. Os tentáculos são sólidos. Existe uma boca central com lábios na extremidade de um curto manúbrio. Os pólipos, quando presentes, apresentam um tubo peridérmico (composto por duas camadas) circundando os tecidos do animal, podendo ser solitários ou coloniais. 
2. Medusas diminutas ligeiramente achatadas; lóbulos marginais com uma mancha pigmentar amarela de posição central Nausithoe aurea*

Medusas diminutas em forma de dedal; lóbulos marginais sem mancha pigmentar (coloração decorrente da presença de zooxantelas) Linuche unguiculata*

Subclasse Discomedusae. As medusas não apresentam sulco coronal. A umbrela pode variar de achatada a hemisférica. Os tentáculos, se presentes, são ocos. Os cantos da boca são alongados e formam braços orais (quatro ou oito). Os pólipos apresentam uma periderme (uma camada) restrita à porção basal do animal.

3. Tentáculos presentes; quatro braços orais distintos; boca central...Semaeostomeae

Tentáculos ausentes; oito braços orais fusionados ao menos na sua porção mais próxima ao disco braquial; com muitas bocas diminutas

Rhizostomeae

Ordem Semaeostomeae. As medusas dessa ordem possuem os cantos da boca bastante alongados formando braços orais, geralmente com as bordas onduladas. Os tentáculos são ocos, e podem se localizar na margem ou na subumbrela. A cavidade gastrovascular é dividida formando bolsas, separadas por septos radiais ou por um sistema de canais. A margem do sino é recortada e pode apresentar oito a dezesseis ropálios.

4. Cavidade gastrovascular sem canais radiais Chrysaora lactea

Cavidade gastrovascular com canais radiais ......... 5

5. Tentáculos inseridos próximo à margem da umbrela; ropálios marginais Aurelia sp.

Tentáculos inseridos distante da margem, na subumbrela; ropálios também localizados na subumbrela Drymonema dalmatinum

Ordem Rhizostomeae. Os quatro braços orais estão divididos formando oito, que apresentam diversas bocas diminutas em sua extensão. As medusas não apresentam tentáculos, e a boca central está ausente. A cavidade gastrovascular é dividida em um complexo sistema de canais. A margem do sino é recortada e pode apresentar oito a dezesseis ropálios.

6. Canal circular ausente ...... Cassiopea xamachana* Canal circular presente.

.................................. 7

7. Escápulas presentes ......... Stomolophus meleagris

Escápulas ausentes ... 8

8. Uma rede de canais anastomosados interna ao canal circular; coloração marrom-amarelada com manchas brancas ........... Phyllorhiza punctata

Apenas três ou quatro canais centrípetos internos ao canal circular e não conectados a ele; coloração esbranquiçada com manchas irregulares marrons ......... Lychnorhiza lucerna

Chave para identificação das espécies de Scyphozoa do Brasil (pólipos).
1. Pólipos com um tubo peridérmico circundando todo o corpo do animal

... 2

Pólipos com um tubo peridérmico restrito à porção basal do corpo do animal

2. Pólipos solitários

Cassiopea xamachana

Pólipos coloniais .................. Linuche unguiculata

Agradecimentos. À Fundação de Amparo à Pesquisa do Estado de São Paulo pela concessão de bolsa de doutorado e pósdoutorado a ACM (FAPESP 99/05374-7 e 03/02433-0); à CAPES pela concessão de bolsa de mestrado a SNS, Pós-Graduação, Área Zoologia, IB USP. Aos Drs H. Matthews-Cascon (UFC), P. A. Horta Jr. (UFSC), L. L. Longo (IB USP), T. M. C. Lotufo (UFC), A. E. Migotto (CEBIMar-USP), F. L. da Silveira (IB USP), D. O Pires (MNRJ), MSc. O. M. P. Oliveira (CEBIMar, IB USP) pelo apoio, facilidades de coleta e acesso a diferentes espécies. Aos Drs M. N. Dawson (UNSW, Austrália), G. Jarms (ZIM, Alemanha) e A. C. Marques (IB USP), e MSc. A. Lindner (Duke University, EUA) pelo acesso a algumas referências. Agradecemos aos autores das fotografias por permitirem o uso das mesmas. Somos gratos ao Dr Fábio L. da Silveira por permitir o uso das instalações e pela leitura crítica do manuscrito. Ao Dr. A. E. Migotto e revisores anônimos pelas sugestões no texto.

\section{REFERÊNCIAS BIBLIOGRÁFICAS}

Agassiz, L. 1862. Contributions to the natural history of the United States of America. IV. Second monograph, in five parts, Acalephs in general, Ctenophorae, Discophorae, Hydroidae, homologies of the Radiata. Boston, Little, Brown \& Co. 380p.

AntipA, G. R. 1892. Eine neue Art von Drymonema. Jenaische Zeitschrift für Naturwissenschaft 27:337-339.

Arai, M. N. 1997. A functional biology of Scyphozoa. London, Chapman \& Hall. 360p.

BARnes, R. D. 1990. Zoologia dos Invertebrados. 4 ed. São Paulo, Roca. 1179p

Bigelow, H. B. 1914. Note on the medusan genus Stomolophus, from San Diego. University of California Publications in Zoology 13(10):239-241.

Bigelow, R. P. 1892. On a new species of Cassiopea from Jamaica. Zoologischer Anzeiger 15:212-214.

Conant, F. S. 1897. The Cubomedusae. Memoirs of the Biological Laboratory, Johns Hopkins University, 4(1):1-61.

Cornelius, P. F. S. 1997a. Class Scyphozoa - jellyfish. In: Richmond, M. D. ed. A guide to the seashores of Eastern Africa and the Western Indian Ocean islands. Stockholm, SIDA/ Department for Research Cooperation, SAREC. p.122-125. 1997b. Keys to the genera of cubomedusae and scyphomedusae (Cnidaria). In: den Hartog, J. C. ed. Proceedings of the 6th International Conference on Coelenterate Biology, 1995. Leiden, Nationaal Natuurhistorisch Museum. p.109122

Dawson, M. N. 2003. Macro-morphological variation among cryptic species of the moon jellyfish, Aurelia (Cnidaria: Scyphozoa). Marine Biology 143(2):369-379.

Dawson, M. N. \& JACoBs, D. A. 2001. Molecular evidence for cryptic species of Aurelia aurita (Cnidaria, Scyphozoa). Biological Bulletin 200(1):92-96.

Dawson, M. N. \& Martin, L. E. 2001. Geographic variation and ecological adaptation in Aurelia (Scyphozoa, Semaeostomeae): some implications from molecular phylogenetics. Hydrobiologia 451:259-273.

Eschscholtz, F. 1829. System der Acalephen. Eine ausführliche Beschreibung aller Medusenartigen Strahltiere. Berlin, Ferdinand Dümmler. 190p.

Ferreira, A. B. DE H. 1986. Novo dicionário da língua portuguesa. 2 ed. Rio de Janeiro, Nova Fronteira. 1838p.

Fewkes, J. W. 1882. Notes on Acalephs from the Tortugas, with a description of new genera and species. Bulletin of the Museum of Comparative Zoology at Harvard College 9(7):251-289. 
Gershwin, L. 1999. Clonal and population variation in jellyfish symmetry. Journal of the Marine Biological Association of the United Kingdom 79:993-1000.

Goy, J. 1979. Campagne de la Calypso au large des côtes atlantiques de l'Amérique du Sud (1961-1962) - 35. Méduses. Résultats scientifiques des campagnes de la Calypso 11:263-296.

Haddad, M. A. 2002. Cnidaria. In: Ribeiro-Costa, C. S. \& Rocha, R. M. coords. Invertebrados. Manual de aulas práticas. Ribeirão Preto, Holos. p.24-50.

Haddad, V., Jr.; Silveira, F. L. da; Cardoso, J. L. C. \& Morandini, A. C. 2002. A report of 49 cases of cnidarian envenoming from Southeastern Brazilian coastal waters. Toxicon 40(10):14451450 .

HaecKel, E. 1880. Das System der Medusen. Erster Theil einer Monographie der medusen. Zweite Hälfte des ersten Theils: System der Acraspeden. Denkschriften der MedizinischNaturwissenschaftlichen Gesellschaft zu Jena 1:361672 .

_. 1882. Report on the deep-sea medusae dredged by H.M.S. Challenger during the years of 1873-1876. Report on the scientific results of the voyage of H.M.S. Challenger during the years 1873-1876. Zoology IV(part II):1-154.

Heeger, T. 1998. Quallen. Gefährliche Schönheiten. Stuttgart, Wissenschaftliche Verlagsgesellschaft mbH. 358p.

Hummelinck, P. W. 1933. Zur kenntnis der ScyphomedusenGattung Cassiopea, mit besonderer Berücksichtigung westindischer Formen. Zoologischer Jahrbucher (Syst.) 64(3/5): $453-502$.

JARMS, G. 1990. Neubeschreibung dreier Arten der Gattung Nausithoe (Coronata, Scyphozoa) sowie Wiederbeschreibung der Art Nausithoe marginata Kölliker, 1853. Mitteilungen aus dem hamburgischen zoologischen Museum und Institut 87:7-39.

1991. Taxonomic characters from the polyp tubes of coronate medusae (Scyphozoa, Coronatae). Hydrobiologia 216-217:463-470

Jarms, G.; Morandini, A. C. \& Silveira, F. L. DA. 2002. Methods and experiences with cultivating polyps and medusae of Coronatae (Cnidaria, Scyphozoa) with a review of important characters. Helgoland Marine Research 56(3):203-210.

KramP, P. L. 1955. A revision of Ernst Haeckel's determinations of a collection of medusae belonging to the Zoological Museum of Copenhagen. Deep-Sea Research 3(Suppl.):149-168.

1961. Synopsis of the medusae of the world. Journal of the Marine Biological Association of the United Kingdom 40: $1-469$

Larson, R. J. 1976. Marine flora and fauna of the Northeastern United States. Cnidaria: Scyphozoa. NOAA Technical Report NMFS Circular 397:1-17.

Leloup, E. 1937. Hydropolypes et scyphopolypes recueillis par C. Dawydoff sur les côtes de l'Indochine française. II. Scyphopolypes. Mémoires de Musée Royal d'Histoire Naturelle de Belgique, Deuxième Série 12:59-70.

Marques, A. C. \& Collins, A. G. 2004. Cladistic analysis of Medusozoa and cnidarian evolution. Invertebrate Biology 123(1):23-42.

Marques, A. C.; Morandini, A. C. \& Migotto, A. E. 2003. Synopsis of knowledge on Cnidaria Medusozoa from Brazil. Biota Neotropica 3(2):1-18.

Mayer, A. G. 1910. The medusae of the world. Volume III. The scyphomedusae. Carnegie Institution of Washington 109:499-735

Mianzan, H. W. 1989a. Las medusas Scyphozoa de la Bahía Blanca, Argentina. Boletim do Instituto Oceanográfico 37(1):29-32.

1989b. Sistematica y zoogeografía de Scyphomedusae en aguas neriticas argentinas. Investigaciones Marinas CICIMAR 4(1):15-34.

Mianzan, H. W. \& Cornelius, P. F. S. 1999. Cubomedusae and Scyphomedusae. In: Boltovskoy, D. ed. South Atlantic Zooplankton, vol. I. Leiden, Backhuys. p.513-559.

Migotto, A. E.; Marques, A. C.; Morandini, A. C. \& Silveira, F. L. DA. 2002. Checklist of the Cnidaria Medusozoa of Brazil. Biota Neotropica 2(1):1-31.

Morandini, A. C. 2003. Deep-sea medusae (Cnidaria: Cubozoa,
Hydrozoa and Scyphozoa) from the coast of Bahia (Western South Atlantic, Brazil). Mitteilungen aus dem hamburgischen zoologischen Museum und Institut 100: $13-25$

Morandini, A. C. \& Silveira, F. L. DA. 2001. New observations and new record of Nausithoe aurea (Scyphozoa, Coronatae). Papéis Avulsos de Zoologia 41(27):519-527.

Morandini, A. C.; Silveira, F. L. Da \& Jarms, G. 2004. The life cycle of Chrysaora lactea Eschscholtz, 1829 (Cnidaria, Scyphozoa, Discomedusae, Semaeostomeae, Pelagiidae) with notes on the scyphistoma stage of three other species. Hydrobiologia 530/531:347-354.

MoreirA, M. G. B. S. 1961. Sôbre Mastigias scintillae sp.nov. (Scyphomedusae, Rhizostomeae) das costas do Brasil. Boletim do Instituto Oceanográfico 11(2):5-30.

Müller, F. 1859. Zwei neue Quallen von Santa Catharina. Tamoya haplonema und quadrumana. Abhandlungen der Naturforschenden Gesellschaft in Halle 5:1-12.

1883. Drymonema an der Küste von Brasilien. Zoologische Anzeiger 6:220-222.

Naumov, D. V. 1961. Stsifoidnye meduzy morei S.S.S.R. [Scyphoid medusae of the seas of S.S.S.R.] [em Russo]. Opredeliteli po Faune S.S.S.R. [Fauna S.S.S.R.] 75:1-98.

OliveirA, L. P. H. 1950. Levantamento biogeográfico da Baía de Guanabara. Memórias do Instituto Oswaldo Cruz 48:363391.

O'Sullivan, D. 1982. A guide to the scyphomedusae of the Southern ocean and adjacent waters. ANARE Research Notes, 4:1-43.

Pantin, C. F. A. \& Dias, M. V. 1952. Rhythm and afterdischarge in medusae. Anais da Academia Brasileira de Ciências 24(4):351-364.

Pastorino, G. 2001. New record of the cubomedusa Tamoya haplonema Muller, 1859 (Cnidaria: Scyphozoa) in the South Atlantic. Bulletin of Marine Science 68(2):357-360.

Purcell, J. E.; Graham, W. M. \& Dumont, H. J. eds. 2001. Jellyfish blooms: ecological and societal importance. Hydrobiologia 451:1-333.

Russell, F. S. 1970. The medusae of the British Isles II. Pelagic Scyphozoa with a supplement to the first volume on hydromedusae. London, Cambridge University. 284p.

RYland, J. S. 1995. Hydroids, sea anemones, jellyfish, and comb jellies. In: Hayward, P. J. \& Ryland, J. S. eds., Handbook of the marine fauna of north-west Europe. New York, Oxford University. p.62-70.

Schroth, W.; Jarms, G.; Streit, B. \& Schierwater, B. 2002. Speciation and phylogeography in the cosmopolitan marine moon jelly, Aurelia sp. BioMed Central Evolutionary Biology 2(1):1-15.

Shangwu, G.; Hueshin, H. \& Shimei, Z. 2002. Fauna Sinica Invertebrata, Vol. 27. Phylum Cnidaria: Class Hydrozoa: Subclass Siphonophorae; Class Scyphomedusae. Beijing, Science. 275p.

ShIH, C. T. 1977. A guide to the jellyfish of Canadian Atlantic waters. Natural History Series 5:1-90.

Silveira, F. L. DA \& Cornelius, P. F. S. 2000. Novas observações sobre medusas (Cnidaria, Scyphozoa, Rhizostomeae) no Nordeste e Sul do Brasil. Acta Biologica Leopoldensia 22(1):9-18.

Silveira, F. L. DA \& Morandini, A. C. 1997. Nausithoe aurea n. sp. (Scyphozoa, Coronatae, Nausithoidae), a species with two pathways of reproduction after strobilation: sexual and asexual. Contributions to Zoology 66(4):235-246.

1998a. Asexual reproduction in Linuche unguiculata (Swartz, 1788) (Scyphozoa: Coronatae) by planuloid formation through strobilation and segmentation. Proceedings of the Biological Society of Washington 111(4):781-794. 1998b. New observations on dormancy mechanisms in Linuche unguiculata (Swartz, 1788) (Scyphozoa: Coronatae). Boletim do Museu Nacional, Nova Série, Zoologia 393:1-7.

Silveira, F. L. DA; Jarms, G.\& Morandini, A. C. 2003. Experiments in nature and laboratory observations with Nausithoe aurea (Scyphozoa: Coronatae) support the concept of perennation by tissue saving and confirm dormancy. Biota Neotropica 2(2): $1-25$ 
Stewart, S.E. 1996. Field behavior of Tripedalia cystophora (Class Cubozoa). In: Lenz, P. H.; Hartline, D. K.; Purcell, J. E. \& Macmillan, D. L. eds. Zooplankton: sensory ecology and physiology. Amsterdam, Gordon \& Breach. p.511-524.

Swartz, O. 1788. Medusa unguiculata och Actinia pusilla. Kungliga Svenska Vetenskaps Akademiens Handlingar 9:198-202.

Tronolone, V. B.; Morandini, A. C. \& Migotto, A. E. 2002. On the occurrence of scyphozoan ephyrae (Cnidaria, Scyphozoa, Semaeostomeae and Rhizostomeae) in the Southeastern Brazilian coast. Biota Neotropica 2(2):1-18.

VANHÖFFEN, E. 1888. Untersuchungen über semäostome und rhizostome Medusen. Bibliotheca Zoologica 1(3):5-52.

1911. Die Medusengattungen Polyclonia und Cassiopea. Sitzungsberichten der Gesellschaft Naturforschender Freunde zu Berlin 7:318-330.

VAnNucci, M. 1951. Hydrozoa e Scyphozoa existentes no Instituto Paulista de Oceanografia. I. Boletim do Instituto Oceanográfico 2(1):67-98.

1954. Hydrozoa e Scyphozoa existentes no Instituto Oceanográfico. II. Boletim do Instituto Oceanográfico 5(1-2):95-149.

. 1957. Distribuição de Scyphozoa nas costas do Brasil. Anais da Academia Brasileira de Ciências 29(4):593-598.

Vannucci-Mendes, M. 1944. Sôbre a larva de Dibothriorhynchus dinoi, sp. n. parasita dos Rhizostomata. (Cestoda Tetrarhynchidea). Arquivos do Museu Paranaense 4:47-82.

von Lendenfeld, R. 1884. The scyphomedusae of the Southern hemisphere. Part III. Proceedings of the Linnean Society of New South Wales 9(2):259-306.

Werner, B. 1983. Die Metamorphose des Polypen von Tripedalia cystophora (Cubozoa, Carybdeidae) in die Meduse. Helgoländer Meeresuntersuchungen 36:257-276.

Werner, B.; Cutress, C. \& Studebaker, J. P. 1971. Life cycle of Tripedalia cystophora Conant (Cubomedusae). Nature 232:582-583.

\section{Glossário}

Abertura bucal diminuta (mouthlet): em Rhizostomeae, uma pequena abertura do sistema gastrovascular em um braço oral, em geral circundada por pequenos tentáculos.

Acraspédota (Acraspedote): termo antigo aplicado a medusas sem véu (Scyphozoa), opondo-se a craspédota (Craspedote), as medusas com véu (Hydrozoa).

Ad-radial (adradial): cada um dos oito raios terciários situados entre um perrádio e um inter-rádio; dividem a umbrela em oito setores iguais e simétricos.

Anastomose (anastomosis): comunicação entre canais, do sistema gastrovascular, que se ramificam e se fundem em diversos graus, diferindo da ramificação simples, onde não há a fusão ou interligação dos canais.

Apêndice (appendage): termo genérico para denominar estrutura anexa ao corpo da medusa. Ex.: apêndice terminal, apêndice do disco oral, etc.

Bolsa gástrica (stomach pouch): divertículo do estômago que se estende até a margem do sino.

Braço oral (mouth arm ou oral arm): estrutura espessada tentaculiforme, ao redor da boca, tipicamente em número de quatro. São projeções dos lábios da boca.

Campo muscular (muscle field): região da subumbrela que apresenta musculatura. Pode ser circular ou radial e estar disposta em áreas determinadas.
Canal circular (circular ou ring canal): canal circundando o perímetro da umbrela, ligando as terminações dos canais radiais.

Canal intermediário (intermediate canal): canal intermediário entre o canal circular (marginal) e o estômago. Também chamado de canal circular intermediário.

Canal inter-ropaliar (interrhopalar canal): um canal radial não no mesmo raio de um ropálio, mas entre os raios ropaliares.

Canal radial (radial canal): canal centrífugo que parte do estômago em direção à periferia (margem) da umbrela. Em algumas espécies alguns canais radiais podem se originar na margem, centrípetos, e terminar em fundo cego sem se ligar ao estômago.

Canal ropaliar (rhopalar canal): canal radial no mesmo raio de um ropálio.

Cálice (calyx): ver cifístoma.

Cifístoma (scyphistoma): estágio de pólipo do ciclo de vida dos cifozoários. Em Discomedusae dividese em disco oral, cálice e pedúnculo.

Ciforriza (scyphorhiza): porção basal do exoesqueleto quitinoso de alguns cifístomas, conectando os indivíduos de uma mesma colônia.

Cifozoário (scyphozoan): diz-se do membro ou espécie da classe Scyphozoa.

Clava (club): um apêndice do braço oral em forma de clava (lateral ou terminal) do braço oral, dele diferindo de modo típico. Em alguns gêneros pode ter formato de remo ou apresentar-se sob a forma circular ou triangular (com 3 lados ou abas).

Coronado (a) (coronate): diz-se do membro ou espécie da ordem Coronatae.

Cubopólipo (cubopolyp): estágio de pólipo do ciclo de vida dos cubozoários.

Cubozoário (cubozoa): diz-se do membro ou espécie da classe Cubozoa.

Disco braquial (arm disc ou mouth arm disc): região da subumbrela onde os braços orais estão inseridos.

Divertículo (diverticulum): ver pedálio.

Éfira (ephyra): medusa imatura de cifozoário liberada a partir de um cifístoma.

Envenenamento (envenomation): processo ou evento onde um organismo injeta veneno em outro. Popularmente denominado de queimadura (sting).

Escapuleta (scapulet, epaulettes): apêndices de algumas Rhizostomeae situados próximos à base (disco braquial) e na porção externa dos braços orais.

Estefanocifístoma (stephanoscyphistoma): estágio de pólipo de membros da ordem Coronatae (Scyphozoa). Também pode ser usado como nome genérico de grupo coletivo (Stephanoscyphistoma Jarms, 1990) em substituição ao gênero Stephanoscyphus Allman, 1874, para designar espécies de pólipos de Coronatae cuja identificação do estágio medusóide fica impossibilitada (ex.: em material preservado).

Estrobilização (strobilation, strobilization): formação de medusas jovens (éfiras), através da formação de discos transversais na porção distal do 
corpo do cifístoma. A estrobilização produz éfiras (medusas imaturas) de forma assexuada. Em geral pode ser denominada polidisco (polidisc), quando ocorre a formação de muitos discos, ou monodisco (monodisc), quando apenas uma éfira é formada por vez. O termo estrobilação também pode ser encontrado nos dicionários.

Exumbrela (exumbrella): veja sino.

Facela (phacellus): ver filamentos gástricos.

Filamento (filament): em algumas Rhizostomeae, uma extensão filamentosa do braço oral, ricamente armada com nematocistos e assumida como tendo uma função defensiva. Pode ser lateral ou terminal.

Filamentos gástricos (gastric filaments, gastric cirri): pequenos filamentos, localizados em grupos ou facelas (phacellae) no contorno do estômago ("facelas" é usado para cubomedusas). Em cifomedusas são, em geral, um pouco mais alongados.

Frênula (frenulum): em cubomedusas, uma das quatro pregas verticais presas à parte interna da abertura do sino e que segura o velário.

Gastrovascular (gastrovascular): o sistema gastrovascular corresponde a um estômago disposto centralmente, bolsas gástricas/estomacais (gastrovascular/stomach pouches), ou canais radiais (radial canals) e, em muitas espécies, às suas anastomoses e o canal circular.

Inter-radial (interradial): cada um dos quatro raios secundários situados entre dois perrádios.

Lábios (lips): pequenas projeções da margem da boca de uma medusa.

Lóbulos (lappets): abas da margem do disco de cifomedusas, de disposição regular, porém variando bastante em forma e número. Lóbulos ropaliares ou oculares (rhopalial ou ocular lappets) são os que ladeiam os ropálios. Em muitas espécies eles diferem em tamanho e forma dos remanescentes, chamados de velares ou interropaliares (velar ou inter-rhopalar).

Manúbrio (manubrium): projeção tubular e central, inserida na superfície da subumbrela e voltada para baixo, portando a boca na extremidade.

Metagênese (metagenesis): alternância de estágios sexuados (medusóides) e assexuados (polipóides) no ciclo de vida.

Octante (octant): porção referente à divisão da umbrela em 8 partes (delimitado pelos ropálios).

Óstio genital, papila genital, pilar, pórtico subgenital (genital ostium, genital papilla, pillar, genital porticus): em medusas rizostomátidas, o óstio genital é o espaço entre as bases de braços orais adjacentes, próximo ao ponto de fusão dos mesmos. A região basal dos braços orais é denominada de pilar, em distinção aos óstios entre eles. Em alguns grupos observa-se a presença de papilas genitais (de formas variáveis), na superfície da subumbrela, parcialmente obstruindo a abertura dos óstios. A cavidade para a qual se abrem os óstios é denominada pórtico subgenital.

Pedálio (pedalium): em cubomedusas, uma extensão da margem da umbrela, em cada um dos cantos, portando tentáculos. Em Chirodropidae eles são palmados, com um tentáculo em cada "dedo" e em
Carybdeidae cada pedálio porta apenas um único tentáculo. Os pedálios apresentam um canal interno que se estende até a ponta do(s) tentáculo(s), sendo uma continuação da cavidade gastrovascular. Em algumas espécies pode ser observada uma projeção deste canal na inserção dos pedálios na umbrela, esta sendo denominada de divertículo.

Pedúnculo (stalk): ver cifístoma.

Periderme (periderm): exoesqueleto quitinoso de um cifístoma.

Perradial (perradial): os perrádios são cada um dos quatro raios primários, na orientação das bolsas estomacais e dos cantos da boca, que dividem a umbrela em quatro setores iguais e simétricos. Entre eles ocorrem os quatro inter-rádios e entre os perrádios e os inter-rádios, os oito ad-rádios.

Plânula (planula): estágio larval dispersivo do ciclo de vida da maioria dos cnidários. Pode ser ciliada ou flagelada e é resultante da fusão dos gametas femininos e masculinos.

Planulóide (planuloid): semelhante a uma plânula, porém resultante de um processo assexuado (ex.: brotamento, segmentação).

Reentrância marginal (marginal cleft): o recorte entre dois lóbulos; reentrância ropaliar (rhopalial cleft) é aquela onde se situa um ropálio.

Rizostomátido (a) (rhizostome): diz-se do membro ou espécie da ordem Rhizostomeae.

Ropálio (rhopalium): nas cubo e cifomedusas o ropálio ou órgão sensorial marginal é uma estrutura tipicamente situada em uma reentrância na margem da umbrela ou em um nicho lateral. Consiste de uma concentração de receptores sensoriais.

Semeostomo (a) (semaeostome): diz-se do membro ou espécie da ordem Semaeostomeae.

Septo gástrico (gastric septum): em Coronatae, um dos quatro septos que fundem a parede do estômago à subumbrela, de posição inter-radial.

Septo radial (radial septum): em cifomedusas, partição interna separando bolsas estomacais.

Sino (bell): corpo da medusa em forma de domo, sem os apêndices. Não existe distinção entre sino e o termo disco (geralmente aplicado para formas mais achatadas); os dois termos geralmente são intercambiáveis quando se descrevem formas intermediárias. O termo umbrela (umbrella) ou umbela (pouco usual) abrange os dois anteriores, sem qualquer significado em relação ao formato do animal. A exumbrela (exumbrella), ou superfície exumbrelar é aquela da superfície aboral do sino, sendo a subumbrela (subumbrella) a superfície oral. O espaço delimitado pelo sino é a cavidade subumbrelar (subumbrellar cavity) preenchida com água e em continuidade com o ambiente. Nas cubomedusas esta ligação se dá pela abertura do velário (velarial aperture).

Subumbrela (subumbrella): ver sino.

Sulco coronal (coronal groove, coronal furrow): conspícuo apenas em medusas de Coronatae, é um sulco circular na superfície exumbrelar de uma medusa. Em muitas espécies delimita diferentes regiões do sino.

Tentáculo (tentacle): apêndice utilizado 
geralmente para apreensão de alimento, situado na margem da umbrela ou na subumbrela, dependendo da espécie; apresenta grande concentração de células urticantes (cnidócitos com nematocistos).

Velário (velarium): em cubomedusas, o velário é um anel de tecido que funciona como um diafragma, diminuindo a abertura da umbrela; apresenta alguns canais chamados de canais velares (velar canals).

Verrugas de nematocistos (nematocyst wart): protuberância de tamanho variável, com nematocistos, encontrada na superfície exumbrelar de medusas; apresentam formas variáveis.

Véu (velum): em hidromedusas, um fino anel de tecido ectodérmico que funciona como um diafragma, diminuindo a abertura da umbrela.

Zooxantela (zooxanthella): organismos unicelulares do grupo dos dinoflagelados que vivem em simbiose com alguns animais (ex.: corais). 